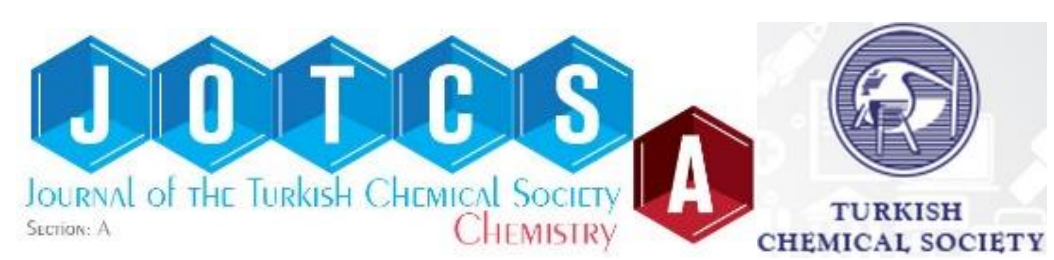

\title{
Preparation and Determination of In Vivo and In Vitro Performance of Doxycycline-Imprinted Contact Lenses for Corneal Neovascularization Treatment
}

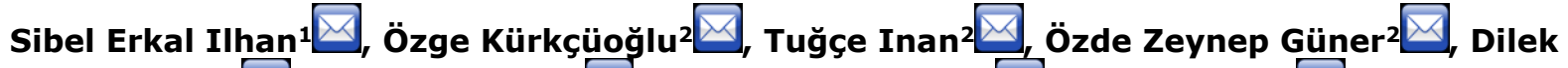 \\ Dalgakıran $^{3 *} \Delta$, Begüm Okutan 4 , Gamze Torun Köse 4 , Ayça Kırmızı 5 , Aylin Okçu \\ Heper $^{5}$, Özlem Gürses 6 , F. Seniha Güner ${ }^{2} * * \square$ \\ ${ }^{1}$ Ankara University, Faculty of Health Sciences, Midwifery Department, 06080 Altındağ Ankara, Turkey \\ 2Istanbul Technical University, Department of Chemical Engineering, Maslak 34469 Istanbul, Turkey \\ ${ }^{3}$ Istanbul Technical University, Polymer Science and Technology, Maslak 34469 Istanbul, Turkey \\ ${ }^{4}$ Yeditepe University, Faculty of Engineering, Department Genetics and Bioengineering, Ataşehir 34755 \\ Istanbul, Turkey \\ ${ }^{5}$ Ankara University, School of Medicine, Department of Medical Pathology, 06100 Sıhhıye Ankara, Turkey \\ ${ }^{6}$ New York Medical College, Department of Microbiology and Immunology, Valhalla, New York, USA \\ *Dilek Dalgakıran's present address is Department of Polymer Engineering, Yalova University, Yalova \\ 77200, Turkey
}

Abstract: The aim of this study is to develop doxycycline imprinted contact lenses that will be used in the treatment of corneal neovascularization, which can eventually cause blindness. For this purpose, doxycycline imprinted contact lenses were first prepared in two different diameters, 5.7 and $5.8 \mathrm{~mm}$, then they were loaded with doxycycline and their in vitro and in vivo performances were determined. In the synthesis of the contact lenses, 2-hydroxyethyl methacrylate was used as a backbone monomer. The functional monomer was selected as itaconic acid using molecular simulations. Doxycycline release profile of the lenses was determined in $\mathrm{NaCl}$ solution at $37^{\circ} \mathrm{C}$. Their doxycycline release was reached about 3 $\mu \mathrm{g} / \mathrm{mg}$ contact lenses in 6 hours. Higuchi model was fitted better than the others as a kinetic model. Swelling degrees of the contact lenses were determined as $38.8 \%$. Cytotoxic response of the lenses was investigated on retinal pigment epithelium cells. According to the results the lenses were not cytotoxic to RPE cell line. In vivo experiments in rat models were performed to study the treatment patterns. The rats were sacrificed fifteen days after treatment, and clinical examination under optical microscope was performed to evaluate neovascularization, infiltration of inflammatory cells, and corneal epithelial changes. In conclusion; doxycycline imprinted contact lenses promise as an effective treatment method for corneal neovascularization.

Keywords: Corneal neovascularization, Contact lens, Doxycycline, Molecular imprinting.

Submitted: May 31, 2018. Accepted: October 01, 2018.

Cite this: Erkal Ilhan S, Kürkçüoğlu Ö, İnan T, Güner ÖZ, Dalgakıran D, Okutan B, Torun Köse G, Kırmızı A, Okçu Heper A, Gürses Ö, Guler FS. Preparation and Determination of In Vivo and In Vitro Performance of Doxycycline-Imprinted Contact Lenses for Corneal Neovascularization Treatment. 2018;5(3):1185-92.

DOI: http://dx.doi.org/10.18596/jotcsa.428846.

\section{**Corresponding author. E-mail: guners@itu.edu.tr}

\section{INTRODUCTION}

Approximately $90 \%$ of ocular diseases are treated with eye drops and ointments, and the remaining with various oral medications. Both concentration and residence time of the drug in the eye are critical for the effective treatment of ophthalmological diseases in general. However, only 1 to $7 \%$ of eye drops is absorbed effectively after the application (1). The remaining of the drug joins the bloodstream leading to low drug bioavailability. In order to assure an effective drug concentration, eye drop should be applied to the patient frequently while watching the toxic concentration limit. When the drug is taken orally, it can cause toxic effect on the gastrointestinal 
system and again the bioavailability can be very low.

Corneal neovascularization (NV) is one of the most common eye diseases, which is characterized by the growth of new blood vessels into the cornea (2). Similar problems mentioned above can be encountered in treatments of corneal NV. Bevacizumab (3), ranibizumab (4) and doxycycline etc. are some topical vascular endothelial growth factor (VEGF) inhibitors used in the treatment of corneal NV (5). Diffusion of the eye drop into deeper levels of the corneal tissue may remain inadequate. On the other hand, treatment with high levels of drug concentration can lead to a toxic effect on the surface of the corneal epithelium in the long term. The necessity of satisfying the indispensable needs in the treatment of corneal NV due to mentioned disadvantages, establishes the basis of this study.

In recent years, scientific researches on contact lenses have been focused on the treatment of ophthalmological diseases $(6,7)$. Hydrogels have been extensively used in the production of contact lenses, due to their high water absorption capacity and high gas permeability. Various methods for drug delivery from hydrogels have recently become available. Controlled drug delivery has been tried to be obtained by practices such as drug absorption in hydrogel lenses from a solution and interjecting colloidal particles into the lenses. Lately, molecularly imprinted hydrogel lenses have been used for improved controlled drug delivery properties (812). In this technique, a complex is formed between a drug and a functional monomer. For obtaining a 3D structure, a cross-linking agent should be used in the synthesis. Drug molecules are removed from the polymer after the completion of polymerization. Thus, some special cavities having complementary in shape, size and chemical functionality to the drug molecule are formed $(7,13)$.

Recently, a series of doxycycline imprinted hydrogels have been prepared from acrylic acid as a functional monomer and ethylene glycol dimethylacrylate as a cross-linker (14). We investigated the reaction kinetics for photo- and thermal polymerization via real-time FTIR spectroscopy and differential photocalorimetry. We found that doxycycline was excited during photopolymerization; thus, the conversion and the overall reaction rate decreased when the template concentration increased in the reaction mixture. After evaluating all results, we chose thermal polymerization method for preparation of doxycycline imprinted hydrogels.

In this study, hydrogel contact lenses were synthesized using molecular imprinting method as in our previous study (14). The active ingredient of the drug chosen for the treatment is doxycycline. The study was realized under three steps; the selection of the functional monomers by a computational method for the synthesis of contact lenses, synthesis and characterization of the contact lenses, and investigation of the biocompatibility of developed contact lenses and their performances during the treatment.

\section{MATERIALS AND METHODS}

\section{Molecular simulations}

Possible functional monomers for the contact lens synthesis were determined as acrylic acid (AA), methacrylic acid (MAA) and itaconic acid (IA). Simulated annealing technique was employed to select a functional monomer for effective imprinting of the drug. $1 \mathrm{~ns}$ long simulations were carried in Materials Studio, using the COMPASS force field. Simulation box under periodic boundary conditions contained drug, functional monomer, backbone monomer 2-hydroxyethyl methacrylate (HEMA) and cross-linker ethylene glycol dimethacrylate (EGDMA) molecules. 25 cycles of annealing were performed between 498 $\mathrm{K}$ and $298 \mathrm{~K}$ to obtain the lowest energy configurations of functional monomers and drugs. The monomer, which had the highest number of hydrogen-bonding interactions from different regions of the drug was selected for synthesis.

\section{EXPERIMENTAL STUDY}

\section{Chemicals}

HEMA was used as a backbone monomer and IA was used as a functional monomer, as was suggested by molecular simulations. Triethylene glycol dimethacrylate (TEGDMA) and 2,2'Azobis(2,4-dimethylvaleronitrile) (Vazoß52) were used as a crosslinking agent and a thermal initiator, respectively. Doxycycline hyclate used as a template molecule was gifted by Deva (Istanbul, Turkey).

\section{Synthesis of Contact Lens}

Molecular imprinted contact lenses were synthesized via free radical polymerization (14). Doxycycline $(0.205 \%$ mole) was dissolved in HEMA ( $92 \%$ mole) during $30 \mathrm{~min}$ in ultrasonic bath below $40{ }^{\circ} \mathrm{C}$. IA ( $3 \%$ mole), TEGDMA ( $5 \%$ mole) and $\operatorname{Vazo} \AA 52$ ( 0.12 by weight \%) were added to the reaction mixture. In order to dissolve the initiator, the reaction mixture was put into ultrasonic bath for $15 \mathrm{~min}$. Because Vazo $\AA 52$ is a low temperature initiator, the temperature of ultrasonic bath was protected under $20^{\circ} \mathrm{C}$. The oxygen in the solution was removed by bubbling nitrogen for $30 \mathrm{~min}$. Then, the reaction mixture was injected into a hydrophobic polymer coated lens mold. The mold was placed into an oven at $45^{\circ} \mathrm{C}$ for $24 \mathrm{~h}$. After synthesis, the contact lenses were removed from the mold and they were immersed in boiling water for 15 minutes to remove unreacted monomers. After that, they were washed in three steps; in $0.01 \mathrm{M}$ oxalic acid:methanol:acetonitrile solution 
(65:15:20), in methanol and in water. Finally, the lenses were dried at $37^{\circ} \mathrm{C}$ for 72 hours in a vacuum oven. Swelling degree of the contact lenses was determined by using equation 1 after they were gently rinsed with distilled water at 37 ${ }^{\circ} \mathrm{C}$ for $48 \mathrm{~h}$.

Swelling degree $(\%)=\frac{\left(W_{2}-W_{1}\right)}{W_{1}} \times 100$

where $W_{1}$ is the weight of the polymer before swelling and $W_{2}$ is the weight of the polymer after swelling.

\section{In Vitro Drug Loading and Release}

For determination of loading capacity of the contact lenses, dried samples were immersed in $75 \mu \mathrm{M}$ DOXH aqueous solutions $(10 \mathrm{~mL})$ and kept at $4{ }^{\circ} \mathrm{C}$ for $24 \mathrm{~h}$, shaken with orbital shaker and protected from light. The initial and final concentrations of the solution were determined by Perkin Elmer Lambda 25 UV/VIS spectrophotometer.

In order to observe release kinetics, the doxycycline imprinted contact lenses were firstly placed into $1 \mathrm{mM}$ doxycycline solution for $48 \mathrm{~h}$ at $4{ }^{\circ} \mathrm{C}$. After loading, samples were washed with distilled water to remove doxycycline hyclate that was absorbed on the surface and then surfaces of the lenses were dried gently by a paper to remove excess water. Each lens was immersed into $10 \mathrm{ml}$ $0.9 \% \mathrm{NaCl}$ solution at $37^{\circ} \mathrm{C}$ with gentle shaking at $100 \mathrm{rpm}$ in amber bottles. Releasing solution $(0.8 \mathrm{~mL})$ was withdrawn at regular intervals and the concentration was detected by the UV/Vis spectrophotometer at $274 \mathrm{~nm}$.

Doxycycline concentration of the loading and release solutions were not same due to limitations of measurements with UV spectrophotometer.

\section{Cytotoxic Response}

Samples were sterilized before adding them to the cell culture. Retinal pigment epithelium cell (RPE cell) line was used for this study. RPE cells ( $2.510^{3}$ cells/well) were seeded in 24 well culture plate. After 6 hours of incubation for the cell attachment, $1 \mathrm{~mL}$ of fresh medium and a piece of contact lens were added into each well, and every week $500 \mu \mathrm{L}$ of fresh medium containing $10 \%$ heat-inactivated fetal bovine serum and 100 units $/ \mathrm{mL}$ streptomycin were added. Cells were incubated at $37{ }^{\circ} \mathrm{C}$ in $5 \% \quad \mathrm{CO}_{2}$ incubator throughout 21 days. There were 4 groups in this study: blank (including medium only), only RPE cells (control), only contact lens, and contact lens with RPE cells. At the end of each time period, XTT (2,3-bis-(2-methoxy-4-nitro-5-sulfophenyl)$2 \mathrm{H}$-tetrazolium-5-carboxanilide, Cell Signaling Technology) was used according to kit protocol. First, the electron coupling (PMS) and XTT labeling reagents were thawed and immediately combined in a $1 \mu \mathrm{L}: 50 \mu \mathrm{L}$ ratio. Then the XTT solution $(500 \mu \mathrm{L})$ was added to the cell culture wells. The absorbance at $450 \mathrm{~nm}$ was determined after 1, 7, 14 and 21 days of cultivation by using Elisa spectrophotometry (Bio-Tek ELx800 Absorbance microplate reader, USA).

\section{In Vivo Study and Histopathological Examination}

Alkaline burn injury model was used in the rat cornea (3). For this purpose, twenty-seven Wistar albino rat corneas injured with $1 \mathrm{~N} \mathrm{NaOH}$ solution were divided into three groups: untreated, eye drop-treated and contact lens-treated groups. The lens treatment was applied during 5 hours per day. Eye drops of doxycycline solution (2 $\mathrm{mg} / \mathrm{mL}$ ) were administered two times daily. The rats were sacrificed fifteen days after treatment.

Corneal specimens were fixed in $10 \%$ buffered formalin for 24 hours. Fixed tissue samples were processed routinely by paraffin embedding technique. Sections of $4 \mu \mathrm{m}$ were obtained and stained with hematoxylin-eosin. The preparations were evaluated under the light microscope by two pathologists who were blinded to the knowledge of groups. Vascularization, infiltration of inflammatory cells, and corneal epithelial changes were examined.

The sections were overviewed for the highest vascular area and the number of blood vessels per square millimeter were counted on the $(10 \times)$ objective of the microscope. The vascularity was recorded as "superficial" if the vascular structures were seen just below the epithelium, superficial stromal, "deep" if the vascular structures were seen deep stromal, above the Descemet's membrane. Inflammatory cells were scored as 0 : no inflammation, 1: perivascular and scattered few inflammatory cells (mainly neutrophils, few lymphocytes) 2: Mild-moderate inflammation 3: moderate inflammation 4: severe inflammation (many diffusely distributed inflammatory cells).

\section{Statistical Analysis}

Statistical analysis was performed with frequency, percentage, standard deviation, Mann Whitney U, and Kruskal Wallis tests.

\section{RESULTS AND DISCUSSION}

\section{Functional Monomer Selection}

Molecular interactions between the drug and functional monomers were revealed by the radial distribution function $g(r)$. This statistical measure represents the probability of finding a specific particle in a shell $\mathrm{dr}$ at a distance $r$ from another particle. A high peak at $r=2 \AA$ indicates hydrogen-bonding between a hydrogen atom and an electronegative atom such as $\mathrm{N}$, $\mathrm{O}$ or $\mathrm{F}$. In Figure $1, g(r)$ values of peaks at $2 \AA$ show that doxycycline molecule made hydrogen-bonding with functional monomers from six different positions. Among the functional monomers, IA made the most stable interactions with the drug from all six positions. In terms of number of 
interactions with the drug, IA was followed by AA then MAA. Therefore, IA acid was used in the contact lens synthesis.

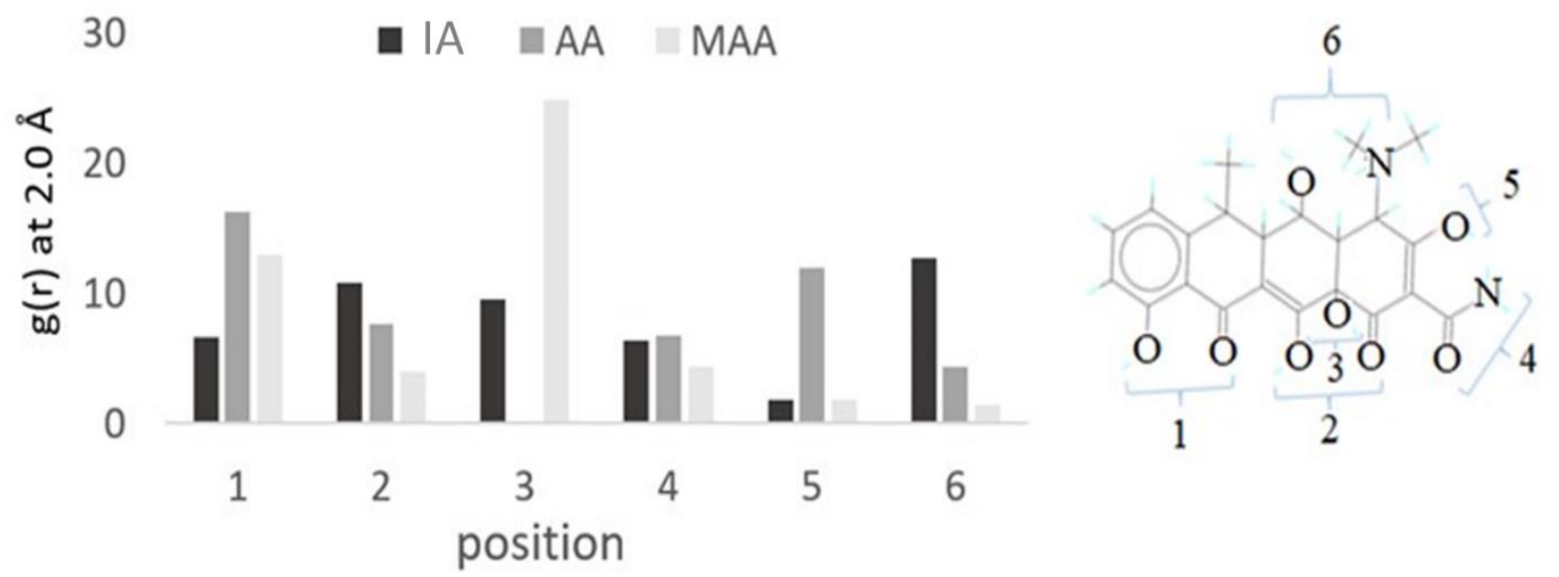

Figure 1. Radial distribution function $g(r)$ results at $r=2 \AA$ for itaconic acid $(\boldsymbol{\square})$, acrylic acid $(\square)$ and methacrylic acid $(\square)$

\section{Synthesis of Contact Lenses}

There were two alternative methods for synthesis of contact lens; photo- and thermal polymerization. We chose thermal polymerization, since doxycycline molecule is sensitive to UV light (14). Teflon mold built for rat's eyes was used in contact lens synthesis, and the contact lenses were prepared in two different diameters (Figure 2). Swelling degrees of the contact lenses were determined as $38.8 \% \pm 2.0$.

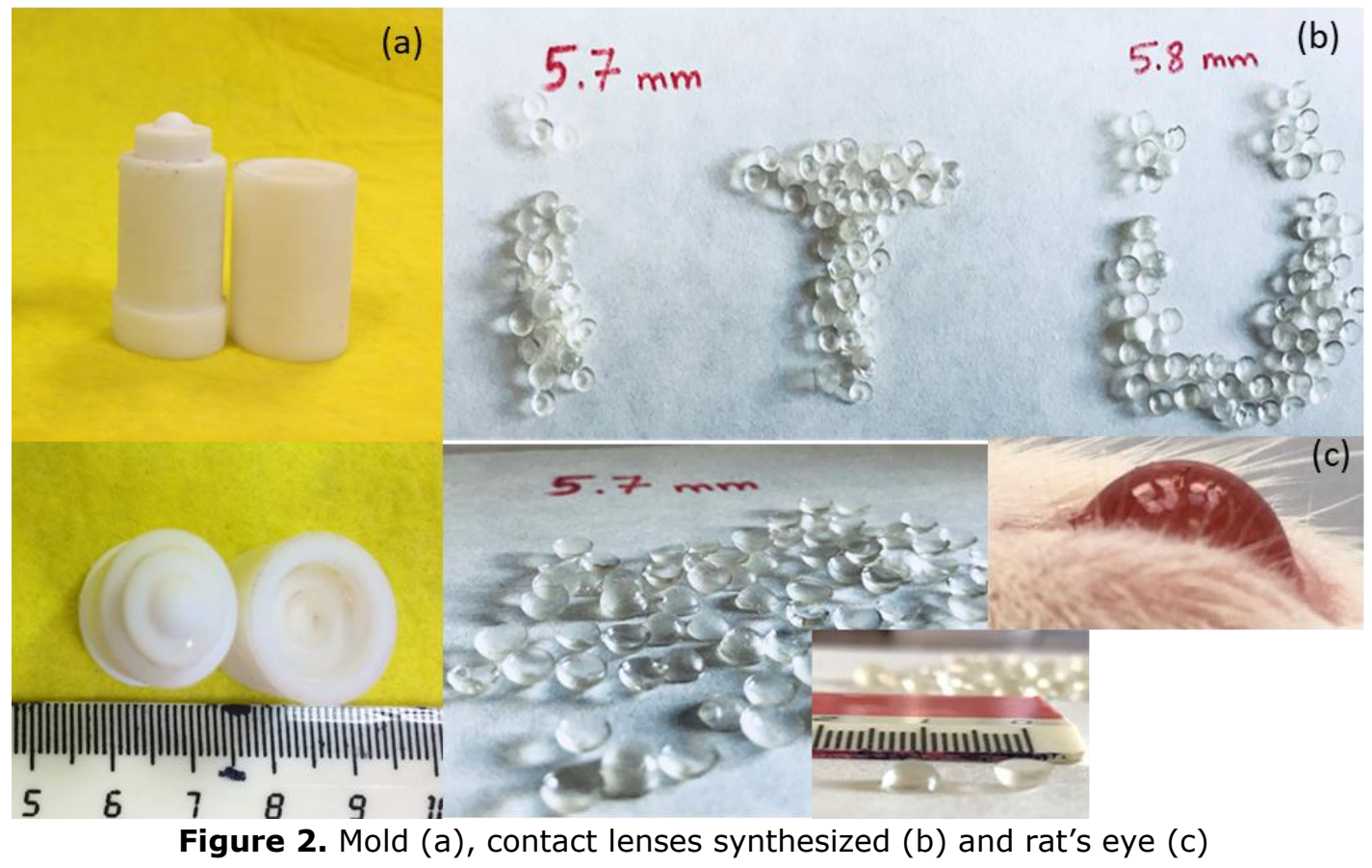

\section{In Vitro Drug Loading and Release}

Doxycycline loading capacity of the contact lenses was found as $0.1805 \pm 0.0100 \mu \mathrm{M}(0.9258 \pm$ $0.0500 \mu \mathrm{g}) \mathrm{drug} / \mathrm{mg}$ contact lens. As explained in the experimental part, loading capacity was investigated in $75 \mu \mathrm{M}$ loading solution. On the other hand, $1 \mathrm{mM}$ doxycycline solution was used for release kinetics. So, the loading and release results cannot be compared.

In order to determine release behavior of the contact lenses, they were loaded with doxycycline in $1 \mathrm{mM}$ doxycycline solution, then their release kinetics were observed in human body conditions which were in $0.9 \% \mathrm{NaCl}$ solution at $37^{\circ} \mathrm{C}$. Obtained results are given in Figure 3. As can be seen, doxycycline release was reached about 3 $\mu \mathrm{g} / \mathrm{mg}$ contact lenses in 6 hours in our study. The mass of a contact lens was measured to be 13.7 $\pm 0.7 \mathrm{mg}$. So, according to our results, doxycycline release was about $41 \mu \mathrm{g}(3 \mu \mathrm{g} / \mathrm{mg} \times$ $13.7 \mathrm{mg}$ ) from each contact lens. In literature, doxycycline concentration for treatment of corneal NV was reported as $37.5 \mu \mathrm{g} /$ day (15). In 
conclusion, in our study doxycycline imprinted contact lenses could be prepared in the recommended dose for treatment of NV.

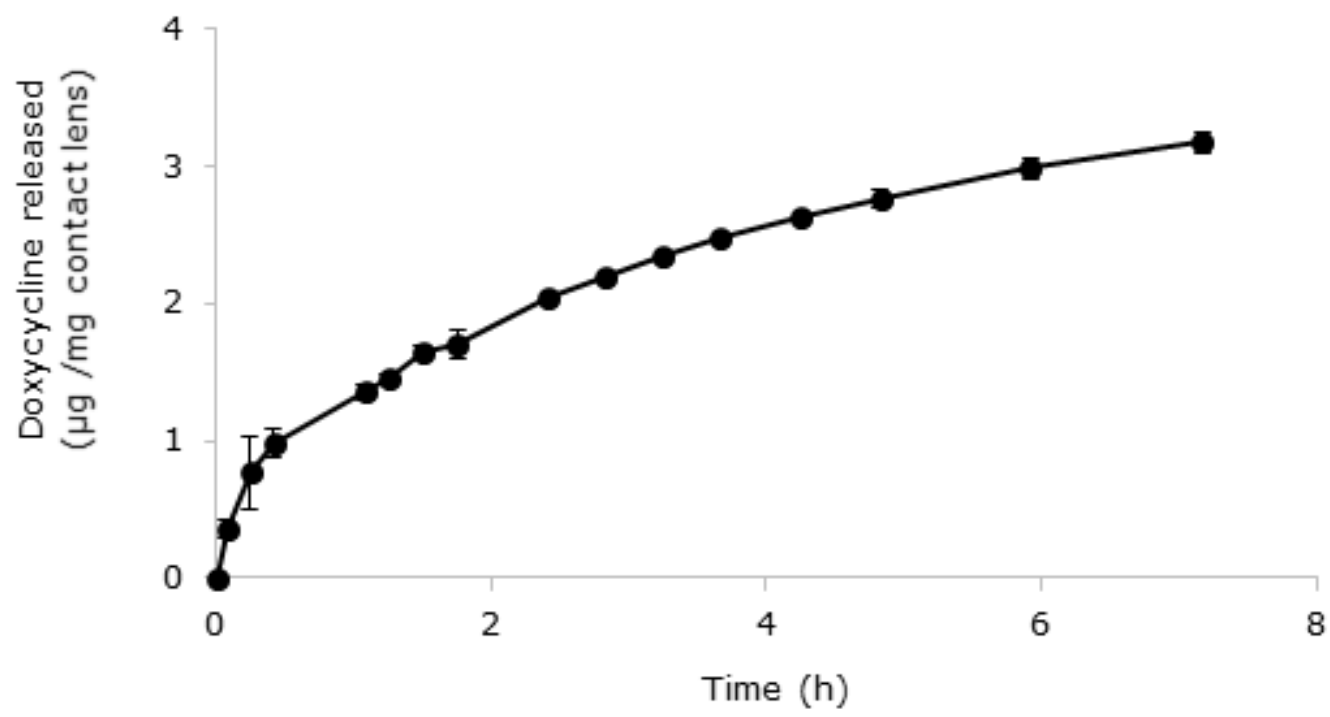

Figure 3. Release profile of doxycycline from contact lens in $\mathrm{NaCl}$ solution at $37^{\circ} \mathrm{C}$.

\section{Cytotoxic Response}

Cell proliferation was observed during 21 days. According to results, the cell viability increased starting from the first day of the cultivation till the end of $7^{\text {th }}$ day in all samples (Figure 4). After 7 days of incubation, cell viability decreased in all samples including only cell (OC). This might be related with the excessive cell proliferation, which led to cell detachment. Therefore, it can be said that this decline was not due to the toxic effect of the contact lens since we can observe the same decrease in all cell containing groups. After 14 and 21 days of incubation, the cell viability increased in all samples again. To conclude, as it can be seen from the results that these lenses were not cytotoxic to RPE cell line.

In this study, zero-order, first-order, Higuchi and Korsmeyer-Peppas models were used to analyze the release of doxycycline from the lenses (Figure 5). Higuchi model was fitted better than the others. This model demonstrates cross-linker effect on imprinted contact lens and it explains drug release based on Fick's law (16). According to the Higuchi model, initial concentration of the drug in the matrix is much higher than drug solubility, drug diffuses only in one dimension, particle size of the drug molecules is much smaller than matrix thickness, and drug diffusivity is constant.

\section{Results of In Vivo Study and Histopathological Examination}

The selected rat's eyes before and after alkaline injury are shown in Figure 6. Our experimental series showed that contact lens treatment had a beneficial effect on the vascular calibers of NV.

Neovascularization, infiltration of inflammatory cells, and corneal epithelial changes were examined in histopathological examination. The obtained results are given in Table 1 for the contact lens-treated, drop-treated and control groups. Histopathological examination was done for all damaged and undamaged eyes. Since the vascular structures were seen just below the epithelium, superficial stromal, all corneas were reported to be superficial. Additionally, no change or epithelial slaughter or necrosis was recorded. According to the results, treatment with doxycycline imprinted contact lenses was effective but not as much as drop treatment.

Our observation during the study was that rats were uncomfortable related to the lens treatment and they were in tendency to remove their lenses. Therefore, we hypothesized that therapeutic effect of the doxycycline imprinted contact lenses was less than expected. We recommended that in vivo study should be repeated and the lens molds should be redesigned for rabbit's eyes. 


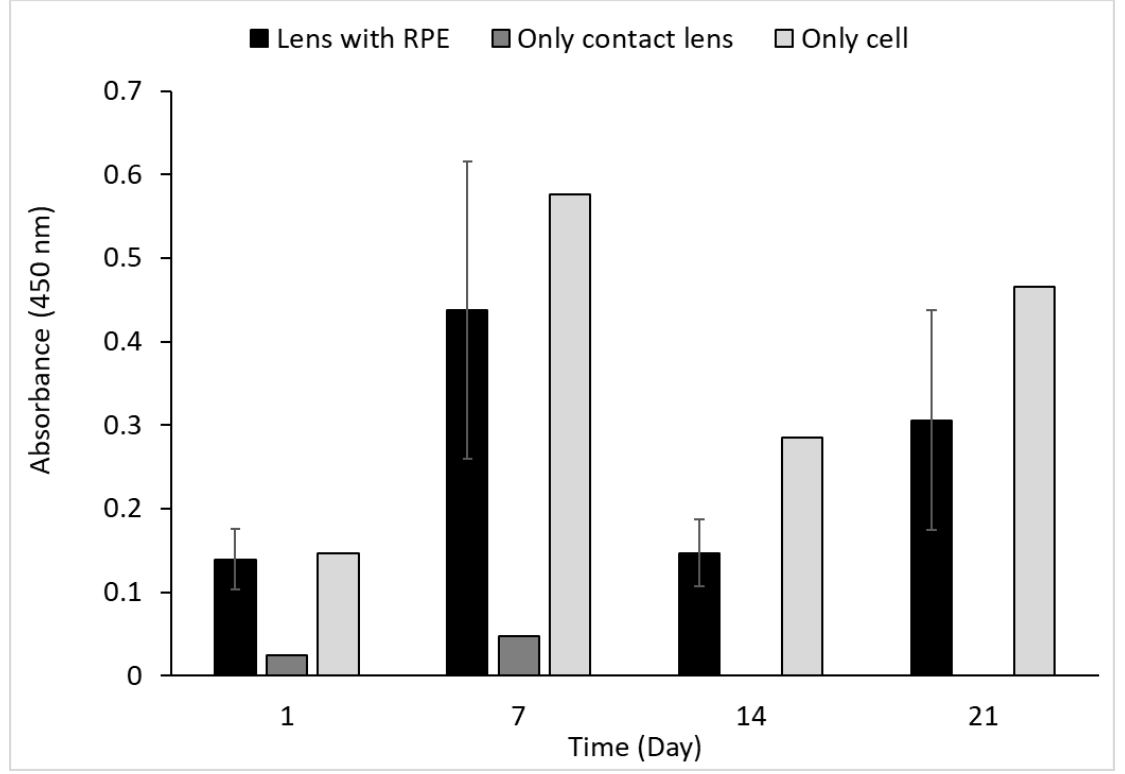

Figure 4. Cytotoxicity of contact lenses (Initial cell seeding density was 2.5103 cells/well. RPE cell proliferation was determined by XTT assay)

Table 1. Vascularization and infiltration of inflammatory cells.

\begin{tabular}{lll}
\hline Sample code $^{*}$ & Vascularization & $\begin{array}{l}\text { Infiltration of } \\
\text { inflammatory cells }\end{array}$ \\
\hline CLT-damaged & $14.3 \pm 4.1$ & $1.5 \pm 1.1$ \\
CLT-undamaged & $2.3 \pm 0.7$ & $1.4 \pm 1.1$ \\
DT-damaged & $3.3 \pm 2.5$ & $0.8 \pm 0.5$ \\
DT-undamaged & $2.0 \pm 0.0$ & $0.3 \pm 0.5$ \\
C-damaged & $18.3 \pm 18.2$ & $1.6 \pm 1.1$ \\
C-undamaged & $2.0 \pm 0.0$ & $1.0 \pm 0.0$ \\
\hline \multicolumn{2}{r}{ Abbreviations: CLT-Contact lens treatment, DT-Drop treatment, C-Control }
\end{tabular}
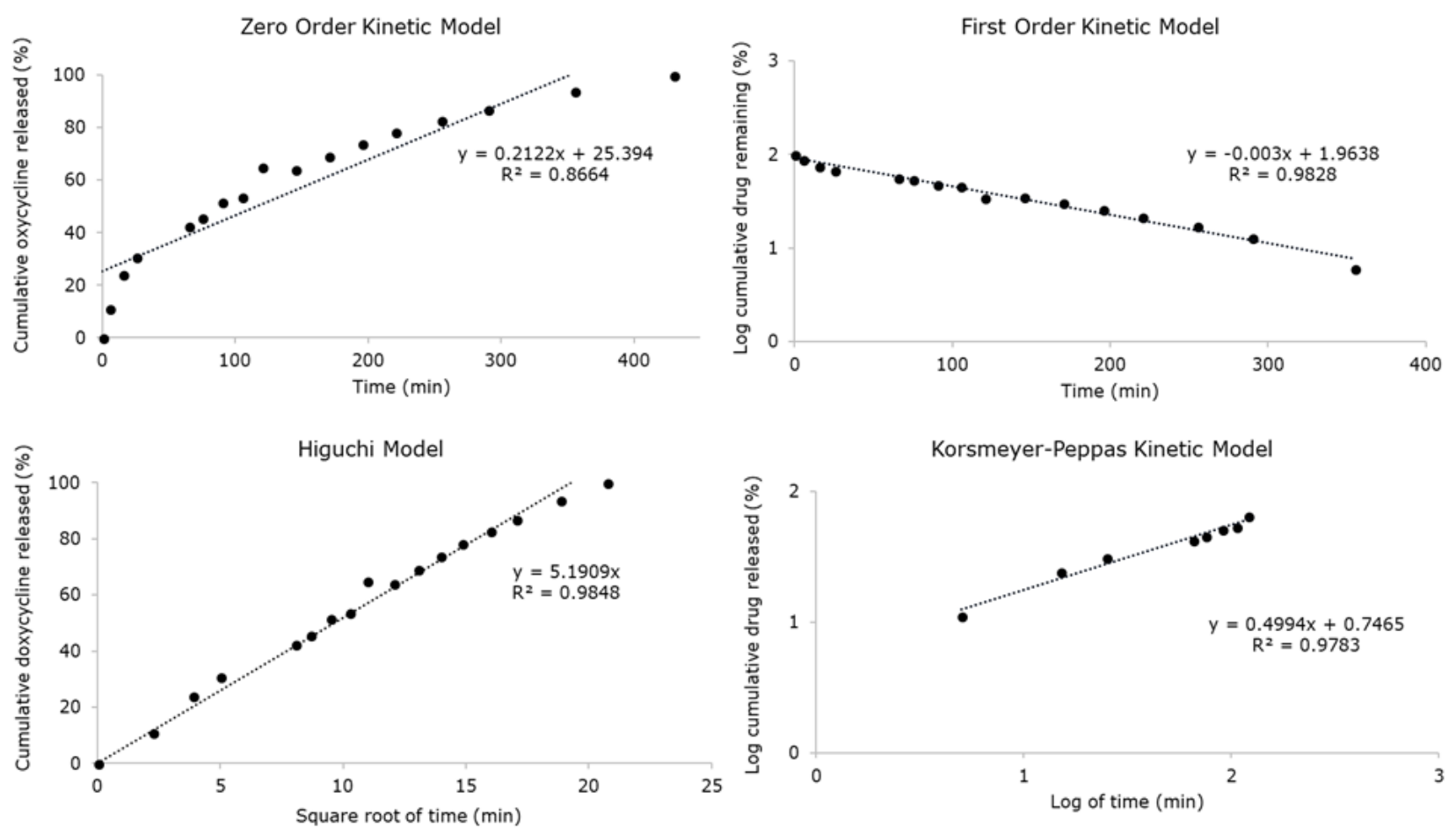

Figure 5. Zero-order, first-order, Higuchi and Korsmeyer-Peppas models for the in vitro release data of doxycycline in $\mathrm{NaCl}$ solution 

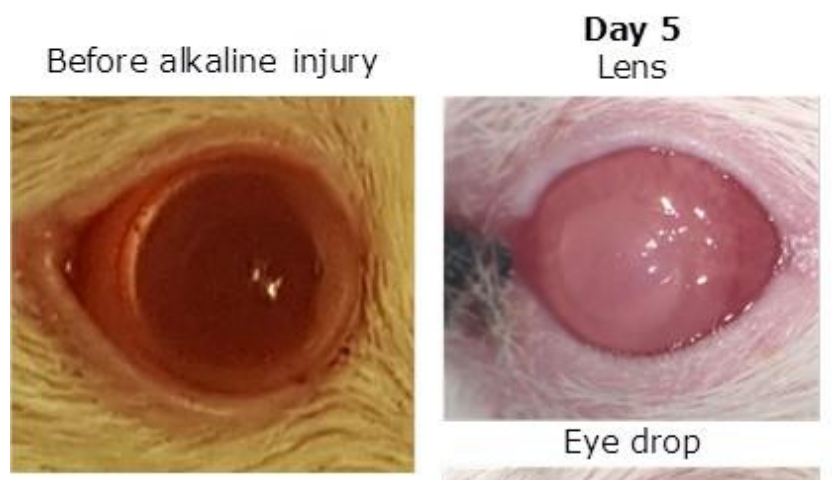

Eye drop

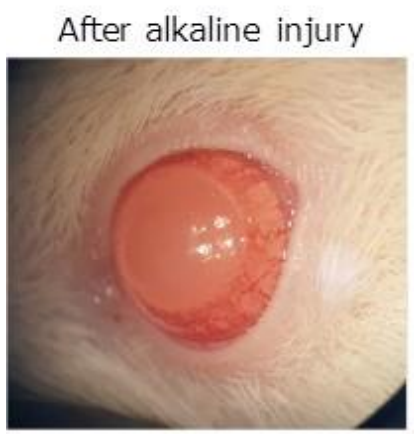

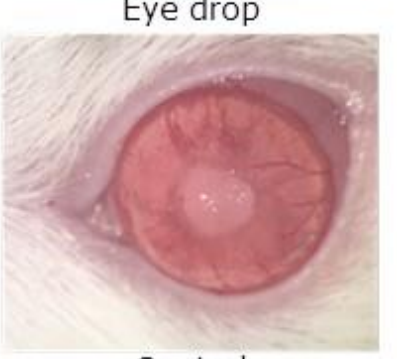

Control

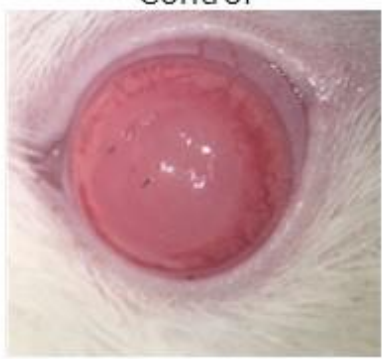

Day 15

Lens

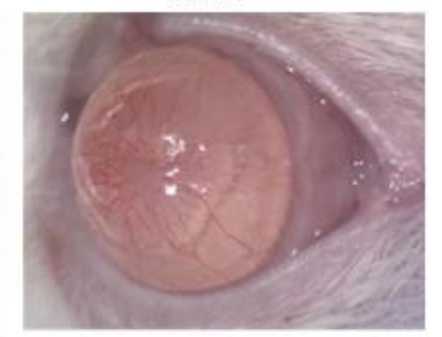

Eye drop

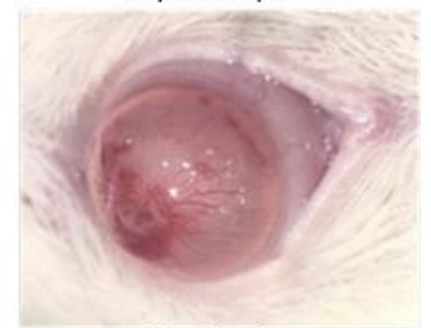

Control

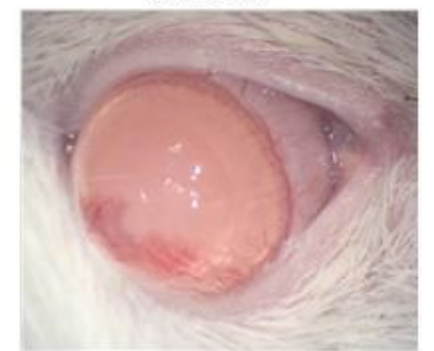

Figure 6. Rat's eyes before and after alkaline injury

\section{Statistical Analysis}

The Kruskal Wallis test was used because there was no homogeneous distribution between groups and the number of groups was less than 30. Corneal vessel count was found to be statistically significant according to Kruskal Wallis test result $(p<0.05)$. Mann-Whitney $U$ test was used to compare the two groups in order to determine the significance between the groups. According to this test, statistically significant results were obtained between the drop group and the control group ( $p=0.038)$, and the number of corneal vessels in the drop-treated group was found to be less. The statistical comparison between the lens-treated group and the drop-treated group was found to be significant $(p<0.001)$, and the number of vessels in the drop-treated group was found to be lower. When the lens-treated group and the control group were statistically compared, no significant results were found ( $p>0.05)$. When the arithmetic average and standard deviation values between these two groups were examined, this result was evaluated as clinically significant when the lens-treated group average was lower and the standard deviation was within the narrow limits.

\section{CONCLUSIONS}

In this study, a new method was described for treatment of corneal neovascularization. First of all, the formulation of doxycycline imprinted contact lens was determined by using computational and experimental methods. All lenses were synthesized with a special mold designed for rat's eye. Their release performances were investigated with four different mathematical models, and Higuchi model fitted data better than the others.

Contact lenses were not cytotoxic to RPE cell line. According to our in vivo results, treatment with contact lens had a beneficial effect on the vascular calibers of neovascularization. The data were reported for the first time in literature.

\section{ACKNOWLEDGMENTS}

This work was funded by The Scientific and Technological Research Council of Turkey (TUBITAK) with grant no. 114M459. The authors would like to especially thank Deva Industry and Trade Inc. (Istanbul, Turkey) for providing doxycycline.

\section{REFERENCES}

1. Aquil M, Gupta H. Contact lenses in ocular therapeutics. Drug Discovery Today. 2012;17(9-10):522-7.

2. Forbes JM, Cooper ME. Mechanisms of diabetic complications. Physiological Reviews. 2013;93:137-88. 
3. Yoeruek E, Ziemssen $F$, Henke-Fahle $S$, Tatar O, Tura A, Grisanti S, Bartz-Schmidt KU, Szurman P. Safety, penetration and efficacy of topically applied bevacizumab: evaluation of eyedrops in corneal neovascularization after chemical burn. Acta Ophthalmologica, 2008;86:322-8.

4. Giulio F, Dastjerdi MH, Okanobo A, Cheng SF, Amparo F, Nallasamy N, Dana R. Topical ranibizumab as a treatment of corneal neovascularization. Cornea. 2013;32(7):992-7.

5. Feizi S, Azari AA, Safapour S. Therapeutic approaches for corneal neovascularization. Eye and Vision. 2017;4:28-38.

6. Byrne ME, Salian V. Molecular imprinting within hydrogels II: Progress and analysis of the field. International Journal of Pharmaceutics. 2008;364:188-212.

7. Cheong WJ, Yang SH, Ali F. Molecular imprinted polymers for separation science: A review of reviews. Journal of Separation Science. 2013;36(3):60928.

8. Magdy Elnashar, editor. Biopolymers. IntechOpen; 2010. Open access peerreviewed chapter 28. Molecularly Imprinted Polymers (PIMs) in Biomedical Applications by Puoci F, Cirillo G, Curcio M, Iemma F, Parisi OI, Spizzirri UG, Picci N. ISBN: 978953-307-109-1.

9. Hu X, Hao, L, Wang, H, Yang $X$, Zhang G, Wang $G$, Zhang $X$. Hydrogel contact lens for extended delivery of ophthalmic drugs. International Journal of Polymer Science. 2011; Article ID 814163.
10. Alverez-Lorenzo C, Yanez F, BarreiroIglesias R, Concheiro A. Imprinted soft contact lenses as norfloxacin delivery systems. Journal of Controlled Release. 2006;113(3)236-44.

11. Garcia DM, Escobar J., Noa Y, Bada N, Hernaez E, Katime I. Timolol maleate release from $\mathrm{pH}$-sensible poly(2hydroxyethyl methacrylate-co-methacrylic acid) Hydrogels. European Polymer Journal. 2004;40(8):1683-90.

12. Tieppo A, White $C$ J, Paine AC, Voyles $M L$, McBride MK, Byrne ME. Sustained in vivo release from imprinted therapeutic contact lenses. Journal of Controlled Release. 2012;157(3):391-7.

13. Vasapollo G, Sole RD, Mergola L, Lazzoi MR, Scardino A, Scorrano S, Mele G.

Molecularly Imprinted Polymers: Present and Future Prospective. International Journal of Molecular Science. 2011; 12:5908-45.

14. Dalgakiran D, Inan T, Güner FS. Investigation of photoinduced polymerization of doxycycline-imprinted hydrogels: effect of template on initiator reactivity, conversion, and reaction rate. Turkish Journal of Chemistry. 2017;41: 862-73.

15. Gordon MK, DeSantis A, Deshmukh M, Lacey CJ, Hahn RA, Beloni J, Anumolu SS, Schlager JJ, Gallo MA, Gerecke DR, Heindel ND, Svoboda KKH, Babin MC, Sinko PJ. Doxycycline Hydrogels as a Potential Therapy for Ocular Vesicant Injury. Journal of Ocular Pharmacology and Therapeutics. 2010;26(5):407-19.

16. Lobo MS, Costa P. Modeling and Comparison of Dissolution Profiles. European Journal of Pharmaceutical Sciences. 2001;13:123-33. 